\title{
Rainwater Harvesting an Instrument to Improve Ground Water Quality and Quantitative Potential: The Case Study of Dwarka Sub-City, Delhi
}

\author{
Prof Gauhar Mahmood* Ms. Ghazala Ishrat** \\ * Prof. Dept. of Civil Engg. Faculty of Engg. \& Tech. Jamia Millia Islamia, Delhi, \\ ** Research Analyst in R\&D Project of Jamia Millia Islamia
}

\begin{abstract}
Dwarka scheme part of the Urban Extension of MPD-2001 of National Capital Territory of Delhi, has been planned to cater to the ever increasing housing requirements of Metropolitan Delhi. Dwarka Sub-City with a total geographical area of $5648 \mathrm{Ha}$ is located in South-Western part of Delhi. The area is envisaged to become a model Sub-city of the 21st century and to house 13 lakhs people. The area is developing fast with more than 400 Group Housing Societies \& 11000 DDA Residential Freehold Flats (in Four and Multistory Buildings), 3100 Residential Plots, housing colonies apart from commercial complexes, District centers, public places etc. The area with present population of 5 lakhs roughly is in severe grip of shortage of the supplied water, as it receives only Rs 2.8 MGD from the concerned water supply agency, against the requirement of 10 $M G D$. In view of this the balance water demand of its residents for domestic \& non-domestic uses is mostly met from the Ground Water. The rampant extraction of Ground Water by Government \& private authorities, has led to the depletion of fresh water layer and decline in the water table at very fast pace in last few years, raising questions about its future availability.

This paper focuses on the methodology of Ground Water Restoration by way of recharging it with the rainwater, which otherwise goes water and leads to flooding / public havoc. The feasibility \& need for implementation of Ground water Recharge Scheme in the area was established through the detailed study \& analysis of the factors governing the Ground Water Discharge \& Recharge in the area: such a Ground water Level behaviour, Ground water Quality, Rainwater quality, Rainfall Intensity \& its distribution, Ground Water \& Rainwater Potential, Aquifer Geometry \& Characteristics and Ground Water requirement of the area. The Depth to Water level measured at different locations in the area was utilized to study the annual \& Pre-Post Monsoon water level fluctuations. Thereafter the impact of Rainwater Harvesting on the Ground Water Quantitative Potential was established based on the mathematical calculations. The study established that the ground water restoration through artificial recharge technique would to some extent help in mitigating water crises in Dwarka Sub-City \& other urban areas.
\end{abstract}

Key words: Rain water harvesting, Ground water \& rainfall potential, Rainfall Intensity, salinity, stage of ground water development, water level, land use pattern.

\section{Introduction}

National Capital Territory of Delhi has experienced a explosive population growth in last few decades on account of natural population growth \& massive in-migration of people from other parts of country. This has resulted into inevitable urbanization. Dwarka Sub-City located in South-West Delhi is part of the Urban Extension of MPD-2001 of National Capital Territory of Delhi and it has been planned to cater to the ever increasing housing requirements of Metropolitan Delhi. With a total area of $5648 \mathrm{Ha}$, Dwarka Sub-City is ultimately meant to house a population of about 1.3 million people. The area encompasses planned / regulated development (1- 29 sectors) and unplanned areas (Housing colonies, villages and Built-up Area), whereas the $1688 \mathrm{Ha}$ is designated as built-up area and the balance $3960 \mathrm{Ha}$ is under the planned / regulated development. Dwarka is developing fast with more than 400 Group Housing Societies \& 11000 DDA Residential Freehold Flats (in Four and Multistory Buildings), 3100 Residential Plots, housing colonies apart from commercial complexes, District centers, public places etc in various sectors. For the present population of

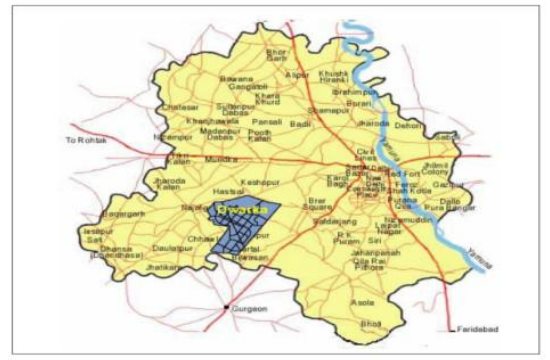
approximately 5 laks the present water demand of the area is 10 MGD and the water received by the area from the concerned water supply agency is approximately only 2.8 MGD thus, forcing its residents to depend to the maximum on Ground Water to fulfill their daily water demand for domestic \& non domestic uses. As a result of which almost all the housing Societies, Private owners etc and some of the DDA colonies have installed tube wells/bore wells to meet their daily water requirement. The rampant extraction of Ground water 
Rainwater Harvesting An Instrument To Improve Ground Water Quality And Quantitative Potential:

has not only resulted in decline in Water table at very fast pace in the area but also degradation in the quality of ground water, as the salinity in Ground Water increases with depth in the area.

\section{Aim of the study:}

The study aims to formulate the strategies to mitigate the water crisis in urban areas of India through Rainwater Harvesting technology and it lays emphasis on in-situ conservation of surplus monsoon runoff for future use i.e. Aquifer storage \& Recovery technique. This besides augmenting the qualitative \& quantitative potential of Ground Water would help in preventing soil erosion \& flooding in urban areas. For the purpose of study Dwarka Sub-city has been taken for reference.

\section{Methodology and Analysis:}

The study primarily involved the in-depth study of the Land Use Master Plan of the area, Field Survey of the entire area \& interaction with the residents, Consultants/Government agencies/NGO's involved in the field, so as to have a wider overview of the problem related to the scarcity of the water and Ground Water in the Sub-city. Thereafter the feasibility \& need for implementation of Ground water Restoration / Recharge Scheme in the area was established through the detailed study \& analysis of the factors affecting Ground Water Discharge \& Recharge in the area such as total water requirement, Ground Water requirement, Ground Water Level behaviour, Ground Water Quality \& its variation with depth, Rainwater quality, Rainfall Intensity \& its distribution, Ground Water \& Rainwater Potential, Aquifer Geometry \& Characteristics and of the area.

The area is underlain by older alluvium and from the study of Tube-well strata charts (obtained from the secondary sources) and interpretation of the Geophysical survey results it was observed that a thick pile of alluvium is overlying the basement rock (located by and large at a depth of $300 \mathrm{~m} \mathrm{b.g.l} \mathrm{in} \mathrm{the} \mathrm{area)} \mathrm{and} \mathrm{it}$ consists of alternate layer of silt, clay, sand and kankar. Nearly fine to medium Sand silt grade sediments occuring frequently up to the depth of $50 \mathrm{~m}$ along with clayey bed admixed with Coarse kankars. On the other hand after the depth of $50 \mathrm{~m}$, silty-clay and clay beds with Kankars increases with depth. Beyond the depth of $100 \mathrm{~m} \mathrm{bgl}$ although the clay beds are common but, the granular zones are not frequent. The presence of frequent clay beds with less frequent granular zone at deeper depth indicated non flushing conditions at deeper depth, resulting in higher concentration of Salinity with depth.

The study of the Ground Water quality \& its variation with depth in Dwarka Sub-city was based on the Physico-Chemical characteristics determined for the Ground Water samples collected at widely spread 35 locations with sources varying in depth from $100 \mathrm{ft}$ to $300 \mathrm{ft}$. The study indicated that the ground water in the area is generally moderately to high saline in nature with Electrical Conductivity more than the potable value of $1000 \mu \mathrm{S} / \mathrm{cm}$ and Total Dissolved Solids above the desirable limit of 500mg/l (although within the permissible limit of $2000 \mathrm{mg} / \mathrm{l}$ ) in almost entire area. It further showed that the Salinity in ground water increases with depth.

The Ground Water Level Behaviour in the area was analyzed by recording depth to Water level at 22 locations (widely spread in the area) during Pre-Monsoon period i.e. May, in the year 2007, 2008 \& 2009 and during Post-Monsoon period i.e. Nov., in the year $2007 \& 2008$.

The observed water levels at different locations are indicated below:

\begin{tabular}{|c|c|c|c|c|c|c|c|c|c|c|c|c|}
\hline $\begin{array}{l}\text { Locatio } \\
\text { n } \\
\text { Sector }\end{array}$ & $\begin{array}{l}\text { Locration } \\
\text { Latitude } \\
\text { Longitrode }\end{array}$ & $\begin{array}{l}\text { G.I } \\
\text { (m) }\end{array}$ & $\begin{array}{c}\text { DTWL } \\
\text { May } \\
07 \\
\text { (mbgl) }\end{array}$ & $\begin{array}{l}\text { RL of } \\
\text { water } \\
\text { table } \\
\text { (m) }\end{array}$ & $\begin{array}{l}\text { DTWL } \\
\text { Nor- } \\
07 \\
\text { (mbgl) }\end{array}$ & $\begin{array}{l}\text { RL of } \\
\text { water } \\
\text { trble } \\
\text { (m) }\end{array}$ & $\begin{array}{l}\text { DTWL } \\
\text { May os } \\
\text { (mbgl) }\end{array}$ & $\begin{array}{l}\text { RL of } \\
\text { water } \\
\text { table } \\
\text { (m) }\end{array}$ & $\begin{array}{l}\text { DTWL } \\
\text { Nor: } \\
\text { 05 } \\
\text { (mbgl) }\end{array}$ & $\begin{array}{l}\text { KL of } \\
\text { water } \\
\text { table } \\
\text { (m) }\end{array}$ & \begin{tabular}{|c} 
DTWL \\
Mlay \\
og \\
(mbgl)
\end{tabular} & $\begin{array}{l}\text { RLof of } \\
\text { water } \\
\text { table } \\
\text { (m) }\end{array}$ \\
\hline $1 / 1$ & $\begin{array}{l}28^{\circ} 35^{\circ} 43.61^{\prime} \mathrm{N} \\
177^{\circ} 04^{\prime} 31.29^{\prime} \mathrm{E}\end{array}$ & 216 & 1191 & 204.09 & 11.09 & 20491 & 12.87 & 203.13 & 12.09 & 20391 & 13.78 & 202.22 \\
\hline $2 / 3$ & $\begin{array}{l}28^{\circ} 36^{\circ} 13.61^{\prime \prime N N} \\
177^{\circ} 02^{\prime} 25.06^{\frac{\mathrm{F}}{\mathrm{E}}}\end{array}$ & 220 & 9.41 & 210.59 & 937 & 210.63 & 10.14 & 209.86 & 994 & 210.06 & 10.54 & 209.46 \\
\hline $3 / 5$ & $\begin{array}{l}28^{\circ} 35^{\circ} 45.81^{\prime N} \mathrm{~N} \\
77^{\circ} 03^{\circ} 1732^{-\mathrm{F}}\end{array}$ & 212 & 10.08 & 20192 & 99 & 202.1 & 12.42 & 19958 & 11.76 & 200.24 & 1291 & 199.09 \\
\hline $4 / 7$ & $\begin{array}{l}28^{\circ} 35^{\circ} 29.75^{\circ} \mathrm{N} \\
177^{\circ} 04^{\prime} 23.97^{\mathrm{E}} \mathrm{E}\end{array}$ & 216 & 1099 & 205.01 & 10.56 & 205.44 & 11.58 & 20442 & 1136 & 204.64 & 11.75 & 20425 \\
\hline $5 / 8$ & $\begin{array}{l}28^{\circ} 34^{\prime 3} 31.73^{\mathrm{N}} \\
177^{\circ} 04^{\circ} 2920^{\prime} \mathrm{E}\end{array}$ & 217 & 11.01 & 20599 & 10.57 & 206.43 & 11.84 & 205.16 & 1093 & 20607 & 1197 & 205.03 \\
\hline $6 / 10$ & $\begin{array}{l}28^{\circ} 34^{\prime} 59.49 \mathrm{~N} \\
777^{\circ} 03^{\prime} \cdot 23.80^{-\mathrm{F}}\end{array}$ & 218 & 9.83 & 20817 & 9.77 & 20823 & 1097 & 207.03 & 10.41 & 20759 & 113 & 2067 \\
\hline $7 / 12$ & $\begin{array}{l}28^{\circ} 35^{\circ} 4758^{\circ N} \\
77^{\circ} 02^{\circ} 39.66^{\mathrm{N}} \mathrm{E}\end{array}$ & 213 & 9.35 & 203.65 & 931 & 203.69 & 1031 & 202.69 & 1132 & 201.68 & 1232 & 200.68 \\
\hline $8 / 12$ & $\begin{array}{l}28^{\circ} 35^{\circ} 36 \mathrm{~N} / \\
77^{\circ} 02 \cdot 28.47 \mathrm{~F}\end{array}$ & 212 & 9.02 & 20298 & 99 & 202.1 & 10.84 & 201.16 & 10.78 & 20122 & 1195 & 200.05 \\
\hline $9 / 13$ & $\begin{array}{l}28^{\circ} 36^{\circ} 03 . \mathrm{A}^{\circ \mathrm{N}} \\
77^{\circ} 02^{\circ} 13.14^{\mathrm{E}} \mathrm{E}\end{array}$ & 215 & 9.11 & 205.89 & 997 & 205.03 & 10.48 & 20452 & 10.73 & 20427 & 11.08 & 20392 \\
\hline $10 / 15$ & $\begin{array}{l}28^{\circ} 36^{\circ} 34.47 \mathrm{~N} \\
177^{\circ} 01^{\circ} 18.03^{-} \mathrm{E}\end{array}$ & 215 & 898 & 206.02 & 9.11 & 205.89 & 9.27 & 205.73 & 991 & 205.09 & 10.01 & 20499 \\
\hline $11 / 16$ & $\begin{array}{l}28^{\circ} 36^{\circ} 23266^{\circ} \mathrm{N} \\
177^{\circ} 00^{\circ} 5727^{\prime} \mathrm{E}\end{array}$ & 216 & 18.08 & 19792 & 17.1 & 1989 & 20.00 & 9600 & 18.04 & 9796 & 18.52 & 97.48 \\
\hline $12 / 16$ & $\begin{array}{l}28^{\circ} 36^{\circ} 01.23^{\circ} \mathrm{N} \\
77^{\circ} 01^{\circ} 05.53^{-} \mathrm{E}\end{array}$ & 213 & 13 & 200 & 12.11 & 200.89 & 12.65 & 20035 & 1297 & 200.03 & 1322 & 199.78 \\
\hline $13 / 17$ & $\begin{array}{l}28^{\circ} 35^{\circ} \cdot 27.18 \% \mathrm{~N} \\
177^{\circ} 01^{\prime} 40.14 \mathrm{E}\end{array}$ & 211 & 11.01 & 19999 & 10.97 & 200.03 & 1159 & 199.41 & 1192 & 19908 & 12.15 & 198.85 \\
\hline $14 / 19$ & $\begin{array}{l}28^{\circ} 34^{\circ} 48.83^{-\mathrm{N}} \\
177^{\circ} 02^{\circ} 53.14^{-} \mathrm{E}\end{array}$ & 214 & 8.83 & 205.17 & 8.71 & 205.29 & 897 & 205.03 & 8.43 & 205.57 & 9.19 & 204.81 \\
\hline $15 / 20$ & $\begin{array}{l}28^{\circ} 34^{\circ} 1526^{\circ} \mathrm{N} \\
177^{\circ} 03^{\circ} 2953^{\mathrm{T}} \mathrm{E}\end{array}$ & 215 & 8.79 & 20621 & 8.78 & 206.22 & 89 & 206.1 & 10.62 & 20438 & 10.34 & 204.66 \\
\hline $16 / 22$ & $\begin{array}{l}28^{\circ} 33^{\circ} \cdot 47.07 \mathrm{~N} \\
77^{\circ} 03^{\circ} \cdot 19.49^{\prime} \mathrm{E}\end{array}$ & 217 & 994 & 207.06 & 991 & 207.09 & 10.37 & 206.63 & 10.18 & 206.82 & 10.87 & 206.13 \\
\hline $17 / 23$ & $\begin{array}{l}28^{\circ} 33^{\circ} 32587 \mathrm{~N} \\
177^{\circ} 03^{\circ} 3456^{\circ} \mathrm{E}\end{array}$ & 221 & 8.72 & 21228 & 8.18 & 212.82 & 8.62 & 21238 & 794 & 213.06 & 8.89 & 212.11 \\
\hline 1823 & $\begin{array}{l}28^{\circ} 33^{\circ} .0731^{\prime 2 N} \\
177^{\circ} 02^{\circ} 37.46^{\mathrm{E}} \mathrm{E}\end{array}$ & 216 & 8.87 & 207.13 & 836 & 207.64 & 9.15 & 206.85 & 894 & 207.06 & 9.45 & 20655 \\
\hline
\end{tabular}


Rainwater Harvesting An Instrument To Improve Ground Water Quality And Quantitative Potential:

\begin{tabular}{|c|c|c|c|c|c|c|c|c|c|c|c|c|}
\hline $19 / 24$ & $\begin{array}{l}28^{\circ} 33^{\prime} 5434^{\prime} \mathrm{N} \\
77^{\circ} 02^{\circ} 0459^{\circ} \mathrm{E}\end{array}$ & 209 & 8.18 & 200.82 & 8.07 & 200.93 & 8.71 & 20029 & 8.01 & 200.99 & 931 & 199.69 \\
\hline $20 / 26$ & $\begin{array}{l}28^{\circ} 32^{\prime} 48.83^{\prime \prime N} \\
777^{\circ} 03^{\prime} 05.46^{\circ} \mathrm{E}\end{array}$ & 217 & 9.75 & 20725 & 927 & 207.73 & 10.32 & 206.68 & 9.67 & 20733 & 10.77 & 206.23 \\
\hline $21 / 28$ & $\begin{array}{l}28^{\circ} 32^{\prime} 44.11^{\prime \prime N} \\
177^{\circ} 01^{\prime} 25.19^{\prime} \mathrm{E}\end{array}$ & 212 & 8.83 & 203.17 & 8.09 & 20391 & 8.99 & 203.01 & 8.75 & 20325 & 9.19 & 02 \\
\hline $\begin{array}{l}\text { 22/Bin } \\
\text { dapur }\end{array}$ & $\begin{array}{l}28^{\circ} 36^{\prime} 2957 \mathrm{MN} \\
177^{\circ} 03^{\prime} 46.44^{\prime} \mathrm{E}\end{array}$ & 214 & 1197 & 202.03 & 11.18 & 20282 & 1297 & 20103 & 1188 & 202.12 & 1399 & 200 \\
\hline
\end{tabular}

Where: G.L Ground Level, DTWL -Depth to Water level in meters below ground level, R.L of Water Table Level above Mean Sea Level in meters

The recorded data was analyzed to obtain ‘Annual \& Pre-Post Monsoon water level fluctuation which are detailed below:

\begin{tabular}{|c|c|c|c|c|c|c|c|c|c|c|}
\hline Sector & $\begin{array}{l}\text { DTWL } \\
\text { (May } \\
07 \text { ) } \\
\text { (mbgl) }\end{array}$ & $\begin{array}{l}\text { DTWL } \\
\text { (Nov. } \\
07 \text { ) } \\
\text { (mbgl) }\end{array}$ & $\begin{array}{l}\text { WLF } \\
\text { (Nov.07- } \\
\text { May07) }\end{array}$ & $\begin{array}{l}\text { DTWL } \\
\text { (May 08) } \\
\text { (mbgl) }\end{array}$ & $\begin{array}{l}\text { DTWL } \\
\text { (Nov. } \\
\text { 08) } \\
\text { (mbgl) }\end{array}$ & $\begin{array}{l}\text { WLF } \\
\text { (Nov.08- } \\
\text { May08) }\end{array}$ & $\begin{array}{l}\text { AWLF } \\
\text { (Nov.08- } \\
07 \text { ) }\end{array}$ & $\begin{array}{l}\text { AWLF } \\
\text { May } \\
08-07)\end{array}$ & $\begin{array}{l}\text { DTWL } \\
\text { (May09) } \\
\text { (mbgl) }\end{array}$ & $\begin{array}{l}\text { AWLF } \\
\text { (May09- } \\
08 \text { ) }\end{array}$ \\
\hline 1 & 11.91 & 11.09 & 0.82 & 12.87 & 12.09 & 0.78 & -1.00 & -0.96 & 13.78 & -0.91 \\
\hline 3 & 9.41 & 9.37 & 0.04 & 10.14 & 9.94 & 0.2 & -0.57 & -0.73 & 10.54 & -0.4 \\
\hline 5 & 10.08 & 9.9 & 0.18 & 12.42 & 11.76 & 0.66 & -1.86 & -2.34 & 12.91 & -0.49 \\
\hline 7 & 10.99 & 10.56 & 0.43 & 11.58 & 11.36 & 0.22 & -0.8 & -0.59 & 11.75 & -0.17 \\
\hline 8 & 11.01 & 10.57 & 0.44 & 11.84 & 10.93 & 0.91 & -0.36 & -0.83 & 11.97 & -0.13 \\
\hline 10 & 9.83 & 9.77 & 0.06 & 10.97 & 10.41 & 0.56 & -0.64 & -1.14 & 11.3 & -0.33 \\
\hline 12 & 9.35 & 9.31 & 0.04 & 10.31 & 11.32 & -1.01 & -2.01 & -0.96 & 12.32 & -2.01 \\
\hline 12 & 9.02 & 9.9 & -0.88 & 10.84 & 10.78 & 0.06 & -0.88 & -1.82 & 11.95 & -1.11 \\
\hline 13 & 9.11 & 9.97 & -0.86 & 10.48 & 10.73 & -0.25 & -0.76 & -1.37 & 11.08 & -0.6 \\
\hline 15 & 8.98 & 9.11 & -0.13 & 9.27 & 9.91 & -0.64 & -0.8 & -0.29 & 10.01 & -0.74 \\
\hline 16 & 18.08 & 17.1 & 0.98 & 20 & 18.04 & 1.96 & -0.94 & -1.92 & 18.52 & 1.48 \\
\hline 16 & 13 & 12.11 & 0.89 & 12.65 & 12.97 & -0.32 & -0.86 & 0.35 & 13.22 & -0.57 \\
\hline 17 & 11.01 & 10.97 & 0.04 & 11.59 & 11.92 & -0.33 & -0.95 & -0.58 & 12.15 & -0.56 \\
\hline 19 & 8.83 & 8.71 & 0.12 & 8.97 & 8.43 & 0.54 & 0.28 & -0.14 & 9.19 & -0.22 \\
\hline 20 & 8.79 & 8.78 & 0.01 & 8.9 & 10.62 & -1.72 & -1.84 & -0.11 & 10.34 & -1.44 \\
\hline 22 & 9.94 & 9.91 & 0.03 & 10.37 & 10.18 & 0.19 & -0.27 & -0.43 & 10.87 & -0.5 \\
\hline 23 & 8.72 & 8.18 & 0.54 & 8.62 & 7.94 & 0.68 & 0.24 & 0.1 & 8.89 & -0.27 \\
\hline 23 & 8.87 & 8.36 & 0.51 & 9.15 & 8.94 & 0.21 & -0.58 & -0.28 & 9.45 & -0.3 \\
\hline 24 & 8.18 & 8.07 & 0.11 & 8.71 & 8.01 & 0.7 & 0.06 & -0.53 & 9.31 & -0.6 \\
\hline 26 & 9.75 & 9.27 & 0.48 & 10.32 & 9.67 & 0.65 & -0.4 & -0.57 & 10.77 & -0.45 \\
\hline 28 & 8.83 & 8.09 & 0.74 & 8.99 & 8.75 & 0.24 & -0.66 & -0.16 & 9.19 & -0.2 \\
\hline Bind & 11.97 & 11.18 & 0.79 & 12.97 & 11.88 & 1.09 & -0.7 & -1.00 & 13.99 & -1.02 \\
\hline
\end{tabular}

Where: DTWL - Depth to Water level in meters , mbgl - Meters below ground level, WLF - Water Level Fluctuation in meters, AWLF - Annual Water Level Fluctuation in meters, ( - ) - Minus sign indicates fall in water table

- $\quad$ Annual Ground Water level fluctuation during the period from May 2007 to May 2008 revealed that the Ground Water at $9.09 \%$ locations showed rise in water table, while Ground Water at $90.91 \%$ showed fall in water table. While the Annual Ground Water level fluctuation during the period from May 2008 to May 2009 indicated that only $4.45 \%$ locations showed in rise in water table \& $95.55 \%$ showed fall in water table presented below:
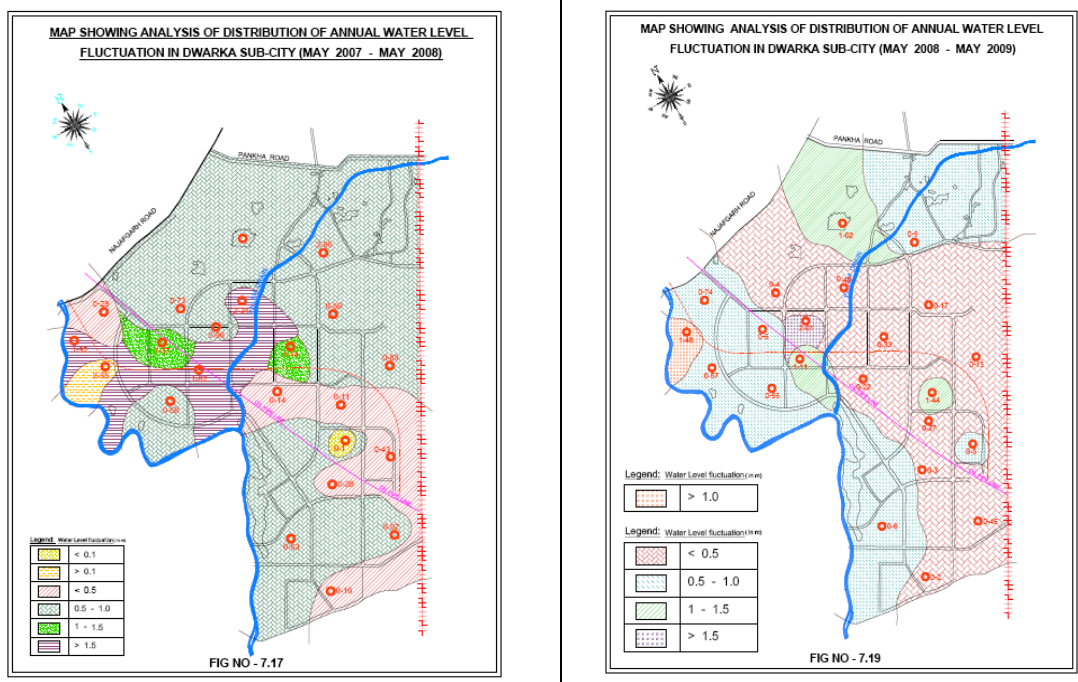
- $\quad$ Pre-Post Monsoon Ground Water Level Fluctuation for the year 2007 showed rise in Ground Water level in $86.36 \%$ locations \& fall in Water table at $13.64 \%$ locations. While, the Pre-Post Monsoon Ground Water Level Fluctuation for the year 2008 showed rise in Ground water level at $77.27 \%$ locations \& fall at $22.73 \%$ locations presented below:

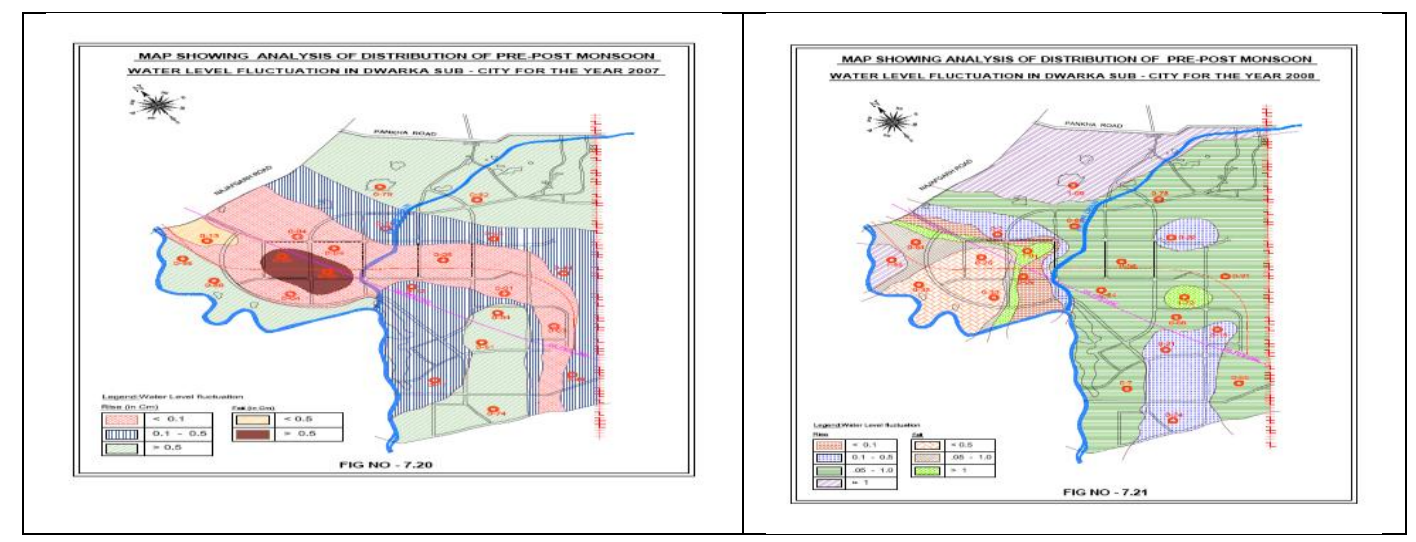

The study established the declining trend of Ground Water in Dwarka Sub-city further it also indicated that the areas where, the rate of natural recharge is not enough to compensate the Ground Water withdrawal rate are increasing with annual rate of decline by an large varying between 0.2 to $1 \mathrm{~m}$.

For the present Research, the 'Ground Water Potential was determined for each sector, by the 'Specific Yield Method' i.e. Using the formula:

Ground Water Potential (Gross Recharge) $=$ Sector Area $\times$ W.L Fluctuation $\times$ Specific yield

Where: Area of each Sector was determined from the Interpretation of Satellite imagery \& lay out Plans for sectors. Water level fluctuation (Pre-Post Monsoon ) for the year 2008 was taken into account. The Recoverable Recharge was taken as $70 \%$ of the Gross Recharge.

The detailed sector-wise analysis of Ground Water Potential for Dwarka Sub-city is presented below:

\begin{tabular}{|c|c|c|c|c|c|}
\hline Sector & Area (Ha) & $\begin{array}{l}\text { Water level } \\
\text { fluctuation }(\mathrm{m} \\
)\end{array}$ & $\begin{array}{l}\text { Specific } \\
\text { Yield }\end{array}$ & $\begin{array}{l}\text { Gross Recharge } \\
\text { (Qgross) }\left(\mathrm{m}^{3} / \text { year }\right)\end{array}$ & $\begin{array}{l}\text { Recoverable Recharge } \\
(\mathrm{I}=70 \% \text { of Qgross }) \\
\left.\left(\mathrm{m}^{3} / \text { year }\right)\right)\end{array}$ \\
\hline 1 & 45.00 & 0.78 & 0.16 & 56160.00 & 39312.00 \\
\hline 2 & 58.38 & 0.78 & 0.16 & 72858.24 & 51000.77 \\
\hline 3 & 158.00 & 0.20 & 0.14 & 44240.00 & 30968.00 \\
\hline 4 & 69.29 & 0.20 & 0.15 & 20787.00 & 14550.90 \\
\hline 5 & 90.14 & 0.66 & 0.16 & 95187.84 & 66631.49 \\
\hline 6 & 93.00 & 0.66 & 0.15 & 92070.00 & 64449.00 \\
\hline 7 & 128.23 & 0.22 & 0.14 & 39494.84 & 27646.39 \\
\hline 8 & 204.83 & 0.91 & 0.17 & 316872.01 & 221810.41 \\
\hline 9 & 110.00 & 0.56 & 0.16 & 98560.00 & 68992.00 \\
\hline 10 & 120.49 & 0.56 & 0.15 & 101211.60 & 70848.12 \\
\hline 11 & 115.00 & 0.56 & 0.16 & 103040.00 & 72128.00 \\
\hline 12 & 119.59 & 1.01 & 0.15 & 181178.85 & 126825.20 \\
\hline 13 & 108.00 & 0.25 & 0.14 & 37800.00 & 26460.00 \\
\hline 14 & 106.00 & 0.25 & 0.15 & 39750.00 & 27825.00 \\
\hline 15 & 115.00 & 0.64 & 0.16 & 117760.00 & 82432.00 \\
\hline 16 & 291.98 & 0.32 & 0.17 & 158837.12 & 111185.98 \\
\hline 17 & 102.00 & 0.33 & 0.15 & 50490.00 & 35343.00 \\
\hline 18 & 90.00 & 0.33 & 0.15 & 44550.00 & 31185.00 \\
\hline 19 & 263.50 & 0.54 & 0.15 & 213435.00 & 149404.50 \\
\hline 20 & 170.40 & 1.72 & 0.18 & 527558.40 & 369290.88 \\
\hline 21 & 158.12 & 0.19 & 0.14 & 42059.92 & 29441.94 \\
\hline 22 & 128.00 & 0.19 & 0.14 & 34048.00 & 23833.60 \\
\hline 23 & 186.00 & 0.68 & 0.16 & 202368.00 & 141657.60 \\
\hline 24 & 275.05 & 0.70 & 0.16 & 308056.00 & 215639.20 \\
\hline 25 & 190.00 & 0.21 & 0.14 & 55860.00 & 39102.00 \\
\hline 26 & 165.00 & 0.65 & 0.16 & 171600.00 & 120120.00 \\
\hline 27 & 73.00 & 0.65 & 0.16 & 75920.00 & 53144.00 \\
\hline 28 & 81.00 & 0.65 & 0.15 & 78975.00 & 55282.50 \\
\hline 29 & 145.00 & 0.24 & 0.14 & 48720.00 & 34104.00 \\
\hline BUA & 1688.00 & 1.09 & 0.17 & 3127864.00 & 2189504.80 \\
\hline \multicolumn{4}{|l|}{ Total = } & 6557311.8 & 4590118.30 \\
\hline
\end{tabular}

BUA-Built-up Area 
Rainwater Harvesting An Instrument To Improve Ground Water Quality And Quantitative Potential:

Since the whole infuse of the study is optimized recharging of ground water reservoir through Rainwater Harvesting system, for dilution of Salinity, hence it involved the detailed study \& analysis of the Rainfall Pattern, its Quality and Rainfall potential. The rainfall pattern in the area was analyzed based on the rainfall data available from Indian Metrological Department, for a sufficiently long period. The distribution of rainfall in the area was found to be non-uniform in time \& space, varying from year to year and mostly concentrated to the monsoon months from July to September. Based on the isohyetal Map of Delhi the annual rainfall in the area was observed to be $600 \mathrm{~mm}$ for the area .The rainwater samples collected during the monsoon month (July) in the year 2007 \& 2008, indicated the variation of Rainwater pH from 6 to 6.5 ( indicating its acidic nature) while TDS was found to be negligible.

Rainfall yearly Potential was determined for each sector considering the four factors of Rainwater Harvesting Scheme ie Roof Top Potential, Road Area Potential, Open area Potential and Total Rainwater Potential. The Rainwater Potential was determined using the formula: Rainfall Potential=A $\times \mathrm{C} \times \mathrm{I}$, where, AArea of the catchment, C-runoff coefficient, I-average annual Rainfall. The runoff coefficients taken for each category of the catchment, for calculation of the Rainfall Potential is as per the prescribed values such as Roof Top- $85 \%$, Road Area-70\% and Open Space/green area-30\%. The Roof Top, Road Area and Open space areas for each sector were determined, on the basis of the study of the Layout Plans of the sectors and interpretation of satellite imagery. The Total rainwater potential is obtained by adding Roof Top Road Area and Open space potential. The detailed Sector-wise analysis of Rainwater Potential for Dwarka Sub-city is presented below:

\begin{tabular}{|c|c|c|c|c|c|c|c|c|}
\hline Sector & $\begin{array}{l}\text { Total Area } \\
\text { (ha) }\end{array}$ & $\begin{array}{l}\text { Art } \\
\text { (ha) }\end{array}$ & $\begin{array}{l}\text { Ard } \\
\text { (ha) }\end{array}$ & $\begin{array}{l}\text { Ao } \\
\text { (ha) }\end{array}$ & $\begin{array}{l}\text { Qrt } \\
\left(\mathrm{m}^{3} / \text { Year }\right)\end{array}$ & $\begin{array}{l}\text { Qrd } \\
\left(\mathrm{m}^{3} / \text { Year }\right)\end{array}$ & $\begin{array}{l}\text { Qo } \\
\left(\mathrm{m}^{3} / \text { Year }\right)\end{array}$ & $\begin{array}{l}\text { Qrain } \\
\left(\mathrm{m}^{3} / \text { Year }\right)\end{array}$ \\
\hline 1 & 45.00 & 18.00 & 9.00 & 18.00 & 91800.00 & 37800.00 & 32400.00 & 162000.00 \\
\hline 2 & 58.38 & 23.35 & 11.68 & 23.35 & 119095.20 & 49039.20 & 42033.60 & 210168.00 \\
\hline 3 & 158.00 & 63.20 & 31.60 & 63.20 & 322320.00 & 132720.00 & 113760.00 & 568800.00 \\
\hline 4 & 69.29 & 27.72 & 13.86 & 27.72 & 141351.60 & 58203.60 & 49888.80 & 249444.00 \\
\hline 5 & 90.14 & 36.06 & 18.03 & 36.06 & 183885.60 & 75717.60 & 64900.80 & 324504.00 \\
\hline 6 & 93.00 & 37.20 & 18.60 & 37.20 & 189720.00 & 78120.00 & 66960.00 & 334800.00 \\
\hline 7 & 128.23 & 51.29 & 25.65 & 51.29 & 261589.20 & 107713.20 & 92325.60 & 461628.00 \\
\hline 8 & 204.83 & 81.93 & 40.97 & 81.93 & 417853.20 & 172057.20 & 147477.60 & 737388.00 \\
\hline 9 & 110.00 & 44.00 & 22.00 & 44.00 & 224400.00 & 92400.00 & 79200.00 & 396000.00 \\
\hline 10 & 120.49 & 48.20 & 24.10 & 48.20 & 245799.60 & 101211.60 & 86752.80 & 433764.00 \\
\hline 11 & 115.00 & 46.00 & 23.00 & 46.00 & 234600.00 & 96600.00 & 82800.00 & 414000.00 \\
\hline 12 & 119.59 & 47.84 & 23.92 & 47.84 & 243963.60 & 100455.60 & 86104.80 & 430524.00 \\
\hline 13 & 108.00 & 43.20 & 21.60 & 43.20 & 220320.00 & 90720.00 & 77760.00 & 388800.00 \\
\hline 14 & 106.00 & 42.40 & 21.20 & 42.40 & 216240.00 & 89040.00 & 76320.00 & 381600.00 \\
\hline 15 & 115.00 & 46.00 & 23.00 & 46.00 & 234600.00 & 96600.00 & 82800.00 & 414000.00 \\
\hline 16 & 291.98 & 116.79 & 58.40 & 116.80 & 595639.20 & 245263.20 & 210225.60 & 1051128.00 \\
\hline 17 & 102.00 & 40.80 & 20.40 & 40.80 & 208080.00 & 85680.00 & 73440.00 & 367200.00 \\
\hline 18 & 90.00 & 36.00 & 18.00 & 36.00 & 183600.00 & 75600.00 & 64800.00 & 324000.00 \\
\hline 19 & 263.50 & 105.40 & 52.70 & 105.40 & 537540.00 & 221340.00 & 189720.00 & 948600.00 \\
\hline 20 & 170.40 & 17.04 & 17.04 & 136.32 & 86904.00 & 71568.00 & 245376.00 & 403848.00 \\
\hline 21 & 158.12 & 47.44 & 79.06 & 31.62 & 241923.60 & 332052.00 & 56923.20 & 630898.80 \\
\hline 22 & 128.00 & 51.20 & 25.60 & 51.20 & 261120.00 & 107520.00 & 92160.00 & 460800.00 \\
\hline 23 & 186.00 & 74.40 & 37.20 & 74.40 & 379440.00 & 156240.00 & 133920.00 & 669600.00 \\
\hline 24 & 275.05 & 82.52 & 55.01 & 137.53 & 420852.00 & 231042.00 & 247554.00 & 899448.00 \\
\hline 25 & 190.00 & 57.00 & 76.00 & 57.00 & 290700.00 & 319200.00 & 102600.00 & 712500.00 \\
\hline 26 & 165.00 & 66.00 & 66.00 & 33.00 & 336600.00 & 277200.00 & 59400.00 & 673200.00 \\
\hline 27 & 73.00 & 29.20 & 14.60 & 29.20 & 148920.00 & 61320.00 & 52560.00 & 262800.00 \\
\hline 28 & 81.00 & 32.40 & 16.20 & 32.40 & 165240.00 & 68040.00 & 58320.00 & 291600.00 \\
\hline 29 & 145.00 & 58.00 & 29.00 & 58.00 & 295800.00 & 121800.00 & 104400.00 & 522000.00 \\
\hline BUA & 1688.00 & 1012.80 & 337.60 & 337.60 & 5165280.00 & 1417920.00 & 607680.00 & 7190880.00 \\
\hline TOTAL & 5648.00 & 2483.38 & 1231.02 & 1933.66 & 12665176.80 & 5170183.00 & 3480562.80 & 21315922.80 \\
\hline
\end{tabular}

\begin{tabular}{|l|l|l|l|l|l|}
\hline Art:- & Area of Roof Top & Ard:- & Road Area & Ao:- & Open Area \\
\hline Qrt:- & Roof Top Rainwater Potential & Qrt:- & $\begin{array}{l}\text { Road Area Rainwater } \\
\text { Potential }\end{array}$ & Qo:- & Open Area Rainwater Potential \\
\hline Qrain:- & \multicolumn{2}{|l|}{ Total Rainwater Potential } & \\
\hline
\end{tabular}

The analysis of the Ground water Potential \& Rainwater Potential of the area indicated that by and large the Roof Top \& Road Area Rainwater Potential of the area is more than the Ground Water Potential. Thus the ideally suitable methodologies of Rainwater Harvesting for the area are Roof Top Rainwater Harvesting \& Total Rainwater Harvesting However in the present study the impact assessment of Total Rainwater harvesting on annual decline rate in Ground Water level \& on Ground Water Potential has been determined based on the mathematical calculations. 
Rainwater Harvesting An Instrument To Improve Ground Water Quality And Quantitative Potential:

The Impact Assessment study of Total Rainwater harvesting on annual decline in Ground Water level is based on the following assumptions:

- Annual Water Level fluctuation for the period May 2009-2008 have been considered for calculations and the areas for which the Annual Water Level fluctuation was not available, the data of surrounding area was taken into account for calculations .

- The impact study has been undertaken considering Total Rainwater Harvesting Potential for each Sector with $30 \%$ loss.

- Ground Water requirement in $\mathrm{m}^{3} / \mathrm{year}$ for each sector of Dwarka Sub-City ( Built-up Area considered as Sector 30) are based on planned population as per zonal Plan of DDA. However, the same has been has been calculated on the following basis:

$>$ For the purpose of calculation of the water requirement of the Dwarka Sub-city, the Per Capita Demand' has been taken as 225 LPCD

$>\quad \%$ Occupancy in Sectors 1-15 taken as $40 \%$, for Sectors 16-19 taken as $30 \%$ and for Sectors $20-29$ taken as $20 \%$ and for Built-up Area as $70 \%$.

$>$ It is assumed that Ground water is required for meeting the water demand for $50 \%$ population of the area

- Nuclear Research Laboratory, IARI has estimated that direct Ground Water Recharge from rainfall infiltration has wide range of spatial and temporal variation, with most parts receiving less than $8 \%$ recharge from rainfall. But on an average only $10 \%$ of the Annual Rainfall is considered as Potential Recharge without any artificial effort Hence, in the present study, It has been assumed that in the absence of artificial recharge efforts, the Natural Recharge to Ground Water would be $=$ Annual rainfall $\times$ Area of Sector $\times 10 \%$

Total recharge to Ground Water $=$ Recharge through natural process + Recharge through artificial efforts. Based on the above assumptions the annual decline obtained after rainwater harvesting with artificial efforts is presented below in the table \& the line Chart.

\section{Impact Assessment Of Rainwater Harvesting On Ground Water Level} Based On Total Rainwater Potential

\begin{tabular}{|c|c|c|c|c|c|c|c|c|}
\hline Sect. & Qrain & $\begin{array}{l}\text { GW } \\
\text { Recharge } \\
\text { Artificially }\end{array}$ & $\begin{array}{l}\text { GW } \\
\text { Recharge } \\
\text { Naturally }\end{array}$ & $\begin{array}{l}\text { Total } \\
\text { Recharge }\end{array}$ & $\begin{array}{l}\text { GW } \\
\text { Withdrawal }\end{array}$ & $\begin{array}{l}\text { Annual } \\
\text { deficit with } \\
\text { RWH }\end{array}$ & $\begin{array}{l}\text { Annual } \\
\text { change in WL } \\
\text { with RWH }\end{array}$ & $\begin{array}{l}\text { Observed } \\
\text { AWLF } \\
\text { May 09-08 }\end{array}$ \\
\hline 1 & 162000 & 113400 & 27000 & 140400.00 & 492750 & -352350.00 & -0.78 & -0.91 \\
\hline 2 & 210168 & 147117.6 & 35028 & 182145.60 & 492750 & -310604.40 & $\begin{array}{l}-0.53 \\
\end{array}$ & $\begin{array}{l}-0.91 \\
\end{array}$ \\
\hline 3 & 568800 & 398160 & 94800 & 492960.00 & 492750 & 210.00 & 0.00 & -0.4 \\
\hline 4 & 249452.4 & 174616.68 & 41574 & 216190.68 & 492750 & -276559.32 & -0.40 & -0.4 \\
\hline 5 & 324504 & 227152.8 & 54084 & 281236.80 & 492750 & -211513.20 & -0.23 & -0.49 \\
\hline 6 & 334800 & 234360 & 55800 & 290160.00 & 492750 & -202590.00 & -0.22 & -0.49 \\
\hline 7 & 461628 & 323139.6 & 76938 & 400077.60 & 492750 & -92672.40 & -0.07 & -0.17 \\
\hline 8 & 737388 & 516171.6 & 122898 & 639069.60 & 492750 & 146319.60 & 0.07 & -0.13 \\
\hline 9 & 396000 & 277200 & 66000 & 343200.00 & 492750 & -149550.00 & -0.14 & -0.13 \\
\hline 10 & 433764.4 & 303635.08 & 72294 & 375929.08 & 492750 & -116820.92 & -0.10 & -0.33 \\
\hline 11 & 414000 & 289800 & 69000 & 358800.00 & 492750 & -133950.00 & -0.12 & -0.33 \\
\hline 12 & 430524 & 301366.8 & 71754 & 373120.80 & 369562.5 & 3558.30 & 0.00 & -2.01 \\
\hline 13 & 388800 & 272160 & 64800 & 336960.00 & 369562.5 & -32602.50 & -0.03 & -0.6 \\
\hline 14 & 381600 & 267120 & 63600 & 330720.00 & 369562.5 & -38842.50 & -0.04 & -0.6 \\
\hline 15 & 414000 & 289800 & 69000 & 358800.00 & 369562.5 & -10762.50 & -0.01 & -0.74 \\
\hline 16 & 1051128 & 735789.6 & 175188 & 910977.60 & 369562.5 & 541415.10 & 0.19 & -0.57 \\
\hline 17 & 367200 & 257040 & 61200 & 318240.00 & 246375 & 71865.00 & 0.07 & -0.57 \\
\hline 18 & 324000 & 226800 & 54000 & 280800.00 & 246375 & 34425.00 & 0.04 & -0.57 \\
\hline 19 & 948600 & 664020 & 158100 & 822120.00 & 349031.25 & 473088.75 & 0.18 & -0.22 \\
\hline 20 & 403848 & 282693.6 & 102240 & 384933.60 & 246375 & 138558.60 & 0.08 & -1.44 \\
\hline 21 & 630898.8 & 441629.16 & 94872 & 536501.16 & 246375 & 290126.16 & 0.18 & -0.5 \\
\hline 22 & 460800 & 322560 & 76800 & 399360.00 & 246375 & 152985.00 & 0.12 & -0.5 \\
\hline 23 & 669600 & 468720 & 111600 & 580320.00 & 349031.25 & 231288.75 & 0.12 & -0.27 \\
\hline 24 & 899448 & 629613.6 & 165030 & 794643.60 & 102656.25 & 691987.35 & 0.25 & -0.6 \\
\hline 25 & 712500 & 498750 & 114000 & 612750.00 & 41062.5 & 571687.50 & 0.30 & -0.6 \\
\hline 26 & 673200 & 471240 & 99000 & 570240.00 & 102656.25 & 467583.75 & 0.28 & -0.45 \\
\hline 27 & 262800 & 183960 & 43800 & 227760.00 & 41062.5 & 186697.50 & 0.26 & -0.45 \\
\hline 28 & 291600 & 204120 & 48600 & 252720.00 & 41062.5 & 211657.50 & 0.26 & -0.2 \\
\hline 29 & 522000 & 365400 & 87000 & 452400.00 & 41062.5 & 411337.50 & 0.28 & $\begin{array}{l}-0.2 \\
\end{array}$ \\
\hline 30 & 7190880 & 5033616 & 1012800 & 6046416.00 & 12811500 & - & -0.40 & -1.02 \\
\hline
\end{tabular}




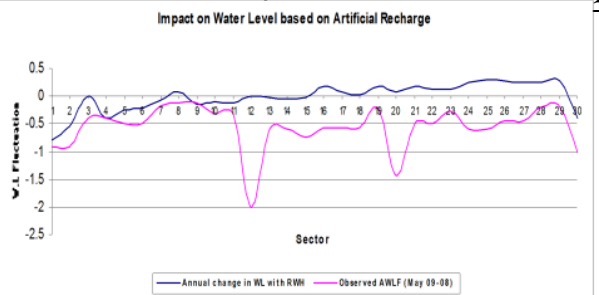

From the above Chart it was observed that with rainwater harvesting the annual decline in water table can be brought down considerably.

The Impact Assessment study of Total Rainwater harvesting on increase in Ground Water Potential is based on the following assumptions:

- It is assumed that the evapo-transpiration losses or any other losses are negligible in nature. Hence Ground Water development is calculated considering that all the Rainfall runoff generated is used to recharge the Ground Water reservoir.

The Ground Water Development after Harvesting was obtained by adding the Rainwater Potential to Gross Recharge / Recoverable Recharge. Based on the above assumptions the \% increase in Gross or Recoverable Recharge obtained is presented in the Table \& Chart below:

\section{IMPACT ASSESSMENT OF RAINWATER HARVESTING GROSS RECHARGE \& RECOVERABLE RECHARGE POTENTIAL BASED ON TOTAL RAINWATER POTENTIAL}

\begin{tabular}{|c|c|c|c|c|c|c|c|}
\hline Sect. & Qrain & $\mathbf{Q}_{\text {Gross }}$ & $\begin{array}{l}\text { G.W.D based on } \\
\text { Gross Recharge }\end{array}$ & $\begin{array}{l}\text { \% inc. in } \\
\mathbf{Q}_{\text {Grossv }}\end{array}$ & $\mathbf{Q}_{\text {Recv }}$ & $\begin{array}{l}\text { G.W.D based on } \\
\text { Recoverable Recharge }\end{array}$ & $\begin{array}{l}\% \text { inc. in } \\
Q_{\text {Recv }}\end{array}$ \\
\hline 1 & 162000 & 56160 & 218160.00 & 288.46 & 39312 & 201312.00 & 412.09 \\
\hline 2 & 210168.00 & 72858.24 & 283026.24 & 288.46 & 51000.768 & 261168.77 & 412.09 \\
\hline 3 & 568800.00 & 44240 & 613040.00 & 1285.71 & 30968 & 599768.00 & 1836.73 \\
\hline 4 & 249452.40 & 20787 & 270239.40 & 1200.04 & 14550.9 & 264003.30 & 1714.34 \\
\hline 5 & 324504.00 & 95187.84 & 419691.84 & 340.91 & 66631.488 & 391135.49 & 487.01 \\
\hline 6 & 334800.00 & 92070 & 426870.00 & 363.64 & 64449 & 399249.00 & 519.48 \\
\hline 7 & 461628.00 & 39494.84 & 501122.84 & 1168.83 & 27646.388 & 489274.39 & 1669.76 \\
\hline 8 & 737388.00 & 316872.01 & 1054260.01 & 232.71 & 221810.41 & 959198.41 & 332.44 \\
\hline 9 & 396000.00 & 98560 & 494560.00 & 401.79 & 68992 & 464992.00 & 573.98 \\
\hline 10 & 433764.40 & 101211.6 & 534976.00 & 428.57 & 70848.12 & 504612.52 & 612.25 \\
\hline 11 & 414000.00 & 103040 & 517040.00 & 401.79 & 72128 & 486128.00 & 573.98 \\
\hline 12 & 430524.00 & 181178.85 & 611702.85 & 237.62 & 126825.19 & 557349.20 & 339.46 \\
\hline 13 & 388800.00 & 37800 & 426600.00 & 1028.57 & 26460 & 415260.00 & 1469.39 \\
\hline 14 & 381600.00 & 39750 & 421350.00 & 960.00 & 27825 & 409425.00 & 1371.43 \\
\hline 15 & 414000.00 & 117760 & 531760.00 & 351.56 & 82432 & 496432.00 & 502.23 \\
\hline 16 & 1051128.0 & 158837.12 & 1209965.12 & 661.76 & 111185.98 & 1162313.98 & 945.38 \\
\hline 17 & 367200.00 & 50490 & 417690.00 & 727.27 & 35343 & 402543.00 & 1038.96 \\
\hline 18 & 324000.00 & 44550 & 368550.00 & 727.27 & 31185 & 355185.00 & 1038.96 \\
\hline 19 & 948600.00 & 213435 & 1162035.00 & 444.44 & 149404.5 & 1098004.50 & 634.92 \\
\hline 20 & 403848.00 & 527558.4 & 931406.40 & 76.55 & 369290.88 & 773138.88 & 109.36 \\
\hline 21 & 630898.80 & 42059.92 & 672958.72 & 1500.00 & 29441.944 & 660340.74 & 2142.86 \\
\hline 22 & 460800.00 & 34048 & 494848.00 & 1353.38 & 23833.6 & 484633.60 & 1933.40 \\
\hline 23 & 669600.00 & 202368 & 871968.00 & 330.88 & 141657.6 & 811257.60 & 472.69 \\
\hline 24 & 899448.00 & 308056 & 1207504.00 & 291.98 & 215639.2 & 1115087.20 & 417.11 \\
\hline 25 & 712500.00 & 55860 & 768360.00 & 1275.51 & 39102 & 751602.00 & 1822.16 \\
\hline 26 & 673200.00 & 171600 & 844800.00 & 392.31 & 120120 & 793320.00 & 560.44 \\
\hline 27 & 262800.00 & 75920 & 338720.00 & 346.15 & 53144 & 315944.00 & 494.51 \\
\hline 28 & 291600.00 & 78975 & 370575.00 & 369.23 & 55282.5 & 346882.50 & 527.47 \\
\hline 29 & 522000.00 & 48720 & 570720.00 & 1071.43 & 34104 & 556104.00 & 1530.61 \\
\hline BUA & 7190880.0 & 3127864 & 10318744.00 & 229.90 & 2189504.8 & 9380384.80 & 328.42 \\
\hline
\end{tabular}

\section{The impact on Ground Water potential:}

- Analysis of 'Ground Water Development' based on Total Rainwater Gross Recharge Potential is presented in Figures below, It is observed that post Harvesting the \% increase in Gross Recharge varies from 76 to $1500 \%$.

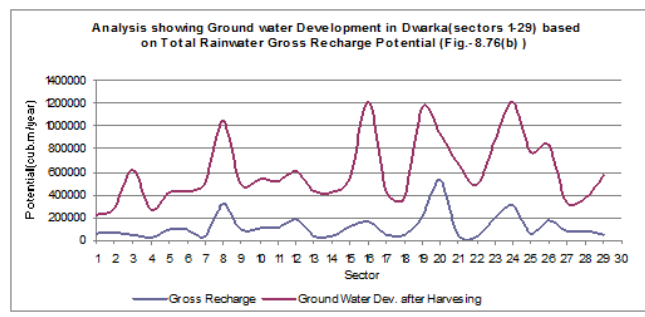



is presented in Figures below, It is observed that post Harvesting the \% increase in Gross Recharge varies from $1094 \%$ to $2142.86 \%$.

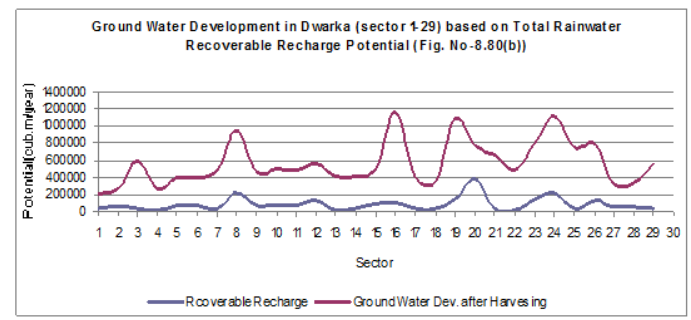

From the above Charts it was established that post rainwater harvesting, there is considerable increase in the quantative potential of the Ground Water .

\section{Conclusion:}

The Dwarka scheme part of the Urban Extension of MPD-2001, has a critical role in Delhi's future development. Today Dwarka-Sub-city may boost of its massive structures, but the fact is that the Sub-city still remains parched. Each day more and more people are making Dwarka Sub-City as their home, as a result of which the Piped water supplied to the Sub-City does not match the demand of its current population thus, forcing its residents to depend to the maximum on Ground Water for meeting their domestic and non -domestic water demand . Besides this, the rainfall in the area is concentrated mostly to few monsoon months and rapid urbanization / increase in paved \& semi-paved areas in Dwarka Sub-city has resulted in very little space as open green areas / unpaved areas for permitting natural recharge to ground water reservoir. The unsustainable Ground water extraction in the area has resulted in decline in water table at alarming rate in last few years and depletion of the fresh water layer. Hence, the provision of potable water to Dwarka Sub-City will be a real challenge in the coming years. To make the water supply system sustainable it is utmost important that the most precious gift of nature i.e. rainwater is harnessed for its beneficial use in most rational manner, by resorting to Rainwater Harvesting. There is need to dovetail the ground water extraction with the ground water recharge in a sustainable manner. The Government has made mandatory requirement for adoption of Rain Water Harvesting System for a Plot area of $100 \mathrm{sqm}$ and above. The Central Ground Water Board (CGWB) also gives emphasis to Roof Top Rain Water Harvesting only . However, this is very small quantity as compared to the withdrawal rate. Hence, this study recommends the adoption of Total rainwater harvesting technique and from present study it has been established that implementation of the Rainwater Harvesting techniques in Urban area is a very attractive and technically feasible technique for Ground water restoration in Urban areas .

\section{References:}

[1]. Rabidyuti Biswas, Deepak Khare and Rama Surbramaniam Shankar, Paper on 'Water Demand Management for an Urban Area : The case Study of Dwarka , a Sub-city of Delhi' Published in Water Utility Management International June 2009.

[2]. K.B.Rajoria, Fellow \& V.K. Sharma Fellow, Paper on" Rainwater Harvesting for Ground Water recharging -A Case Study' Vol. 88, Feb.2008, IE(I) Journal-CV (Page 48-60)

[3]. B.N Maleswara Rao, Paper on' Preparation of Action Plan for Water Resources Management using GIS- Case Study' Presented in 'Hydro 2008'.

[4]. Delhi Development Authority, “Draft Zonal Development Plan, K-II, Dwarka ” dated 30.10.2007 (Page 1-17)

[5]. Central Ground Water Board, Ministry of Water Resources, Government of India, Technical Report (September 2007) "Manual on Artificial recharge of Ground Water' (Page 41-59).

[6]. Central Ground Water Board, Faridabad (Ministry of Water Resources, Government of India), Technical Report "Rainwater Harvesting Techniques to augment Ground water' (Page 4-12).

[7]. Delhi Development Authority, "Zonal Development Plan, Zone -K (Part) Dwarka Sub-city” dated 7.8.2006 (Page1-9)

[8]. Central Ground Water Board ( Sept..2006) “Ground Water Year book 2005-2006, NCT Delhi”(Page 9-31)

[9]. Central Ground Water Board ( Feb.2006) “Hydrogeological Framework \& Groundwater Management Plan of NCT Delhi”(Page 1$5,12-38,61-62)$

[10]. Peter Dillona et al, February 2006, Role of Aquifer Storage in Water Reuse, Desalination Vol. 188, issues 1-3,5 pages 123-134

[11]. Pandey et al, 10July 2003, “Rainwater Harvesting as an adaptation to Climate Change”, Current Science Vol 85 No.1.

[12]. Central Ground Water Board ( Sept. 2000) "Rainwater Harvesting \& Artificial recharge to Ground Water - A Guide to follow"(Page 1-3)

[13]. Water Resources \& Central pollution Control Board, Ministry of Environment \& Forest, Government of India, Technical Report (January 2000) "Status of Ground Water Quality \& Pollution Aspects in National Capital Territory of Delhi'. (Page 68-70)

[14]. Sharma ,S.K and Jain, SK, proceedings of the International Conference on 'Management of Drinking Water Resources', Central Leather Research Institute, Anna Univ. \& Tamil NAdu Water Supply \& Drainage Board, Chennai, India 1997 (Page 129-138)

[15]. KAranath,K.P. ' Ground Water, Assessment, Development \& Management, Ttttttata McGraw-Hill Publ. Co. Ltd, New Delhi, 1997 ( Page 720-721)

[16]. G.S. Birdie "Water Supply \& Sanitary Engineering" ed. 4th (Page 82-83)

[17]. Marino, .A.(1974), 'Water Table fluctuations in response to recharge" American Society of Civil engineers, Journal of Irrigation \& Drainage Division (Page 117-125)

[18]. Geological Survey of India, a Technical Report' Contributions of Geological Survey of India in Delhi Area - A Resume' May 1997 (Page 3,10) 